\title{
Magnetically controlled gravity for protein crystal growth
}

M. C. R. Heijna and P. W. G. PoodtK. TsukamotoW. J. de GripP. C. M. Christianen and J. C. MaanJ. L. A. Hendrix, W. J. P. van Enckevort, and E. Vlieg

Citation: Appl. Phys. Lett. 90, 264105 (2007); doi: 10.1063/1.2752718

View online: http://dx.doi.org/10.1063/1.2752718

View Table of Contents: http://aip.scitation.org/toc/apl/90/26

Published by the American Institute of Physics

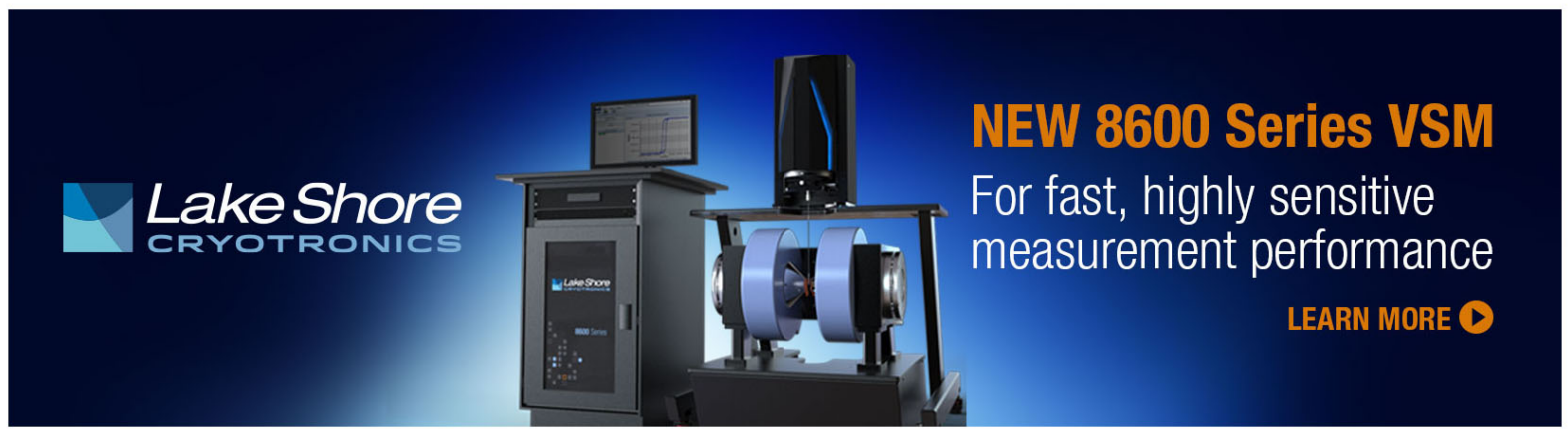




\title{
Magnetically controlled gravity for protein crystal growth
}

\author{
M. C. R. Heijna and P. W. G. Poodt \\ IMM, Solid State Chemistry and High Field Magnet Laboratory HFML, Radboud University Nijmegen, \\ Toernooiveld 1, 6525 ED Nijmegen, The Netherlands \\ K. Tsukamoto \\ Faculty of Science, Department of Earth and Planetary Material Science, Tohoku University, \\ Sendai 980-8578, Japan \\ W. J. de Grip \\ Department of Biochemistry, NCMLS, Radboud University Medical Centre, P.O. Box 9101, \\ 6500 HB Nijmegen, The Netherlands \\ P. C. M. Christianen and J. C. Maan \\ IMM, High Field Magnet Laboratory HFML, Radboud University Nijmegen, Toernooiveld 7 , \\ 6525 ED Nijmegen, The Netherlands
}

J. L. A. Hendrix, W. J. P. van Enckevort, and E. Vlieg ${ }^{\text {a) }}$

IMM, Solid State Chemistry, Radboud University Nijmegen, Toernooiveld 1, 6525 ED Nijmegen, The Netherlands

(Received 6 March 2007; accepted 27 May 2007; published online 29 June 2007)

\begin{abstract}
The occurrence of convective flows during crystal growth is believed to adversely affect crystal quality. Space-based crystal growth is therefore actively pursued, particularly for protein crystals, because buoyancy-driven convection is suppressed in microgravity. Here the authors demonstrate that magnetic fields can be used to tune the effective gravity from 1 to $-0.15 \mathrm{~g}$ during the growth of diamagnetic lysozyme crystals and that convection can be damped, stopped, and even reversed. The growth velocity is strongly reduced in simulated microgravity. This method provides a versatile and accessible way to realize an earth-based tunable gravity environment for crystal growth in which protein crystal quality may be optimized. (ㅇ 2007 American Institute of Physics.
\end{abstract}

[DOI: $10.1063 / 1.2752718]$

On earth, crystal growth from a supersaturated solution is accompanied by buoyancy-driven convection in the liquid, an effect often detrimental to crystal quality. For protein crystals a high quality is required for x-ray structure determination at high resolution, ${ }^{1,2}$ which is of great biotechnological and pharmacological importance. In order to avoid the adverse effects of convection, much effort has been put in examining the virtues of space-based microgravity for protein crystal growth. ${ }^{1-3}$ However, whether zero gravity is the ideal growth condition is still an open question.

It has been shown that gradient magnetic fields can influence convective flows in paramagnetic fluids, ${ }^{4-6}$ and to apply the same approach to seemingly nonmagnetic proteins is appealing, since in fact all diamagnetic materials can be magnetically levitated. ${ }^{7,8}$ A number of experiments have been performed on protein crystal growth under levitation conditions. ${ }^{9,10}$ The criterion for damping convection during crystal growth is, however, quite different from that for levitation, because it relies on balancing buoyancy rather than gravitational force.,

A growing crystal extracts solute from the solution and thus locally reduces the mass density of the solution. The diluted liquid close to the crystal surface will rise due to buoyancy, which leads to a convection pattern, comprising a thin (typically $0.1-0.3 \mathrm{~mm}$ ) laminar flow boundary layer (depletion zone) and a so-called growth plume ${ }^{11}$ on top of the crystal [Fig. 1(a)]. Without convection this plume disappears, diffusion remains the sole means of mass transport, and the depletion zone will expand to infinity [Fig. 1(b)]. To

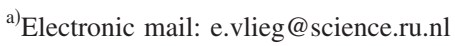

suppress convection the buoyancy forces caused by differences in mass density have to be opposed by magnetic buoyancy forces due to differences in magnetic susceptibility.

For small variations in concentration of the solute, both the density and the susceptibility depend linearly on concentration, i.e., $\rho(c)=\alpha c+\rho_{0}$ and $\chi(c)=\beta c+\chi_{0}$. The convection is then suppressed if

$$
B_{z} B_{z}^{\prime}=\frac{\alpha}{\beta} \mu_{0} g,
$$

where $B_{z}$ is the vertical magnetic field, $B_{z}^{\prime}$ its gradient, and $\mu_{0}$ the magnetic susceptibility. The suppression of buoyancy therefore depends on the concentration dependence of the mass density and susceptibility ( $\alpha$ and $\beta$ ) and not on the mass density and susceptibility themselves, as for normal and magneto-Archimedes ${ }^{12,13}$ levitation.

We demonstrate our method using the diamagnetic protein hen egg-white lysozyme (HEWL) for which crystallization conditions have been well established. ${ }^{14}$ The experiments were performed in a $33 \mathrm{~T}$ water-cooled resistive magnet with a bore diameter of $32 \mathrm{~mm}$ at the High Field Magnet Laboratory at the Radboud University Nijmegen. The magnet, fitted with a double-walled tube for temperature control, contains a shadowgraphy ${ }^{15}$ setup for imaging convection patterns around the growing crystal [Fig. 2(a)]. A glass cuvette (inner dimensions of $8 \times 4 \times 18 \mathrm{~mm}^{3}$ ) with crystal and solution was placed at the position of maximum field gradient [red curve in Fig. 2(b)]. The cuvette is illuminated from the side by a highly collimated beam of light from a halogen lamp, using an optical fiber in combination with a lens and a $75 \mu \mathrm{m}$ pinhole, leading to an image on a 


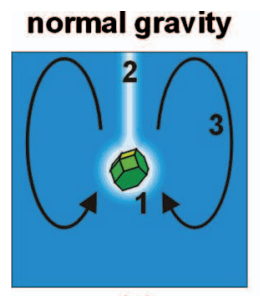

(a)

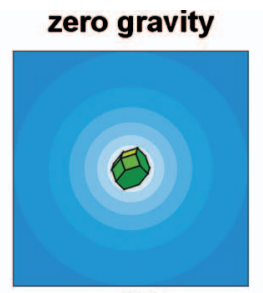

(b)

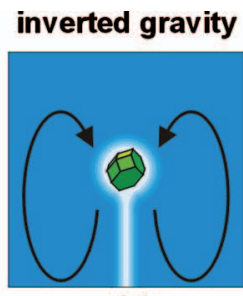

(c)

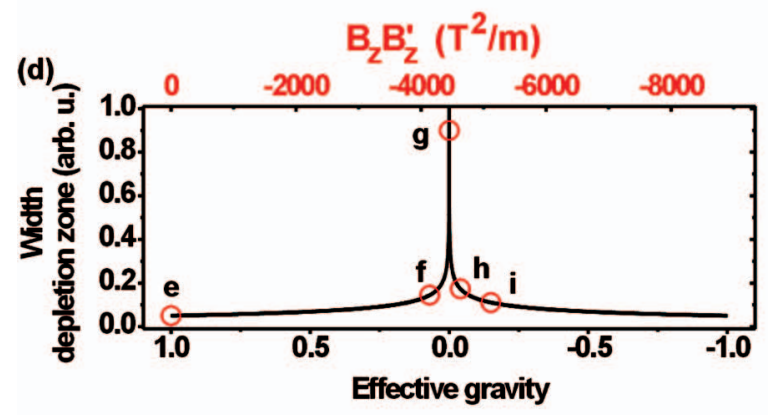

(e)

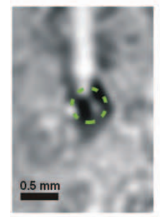

(f)

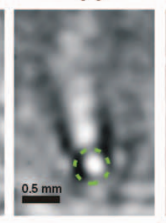

$\begin{array}{lll}B_{0} & 0 & 26.0\end{array}$

$\begin{array}{lll}G_{\text {eff }} & 1 & 0.07\end{array}$
$B_{Z} B^{\prime} z \quad 0 \quad-4130$ (g)

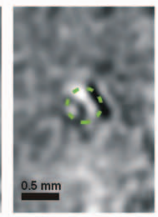

27.0

$-4450$

0 (h)

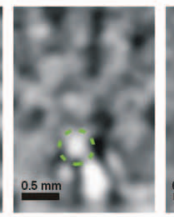

27.5

$-4630$

$-0.04$ (i)

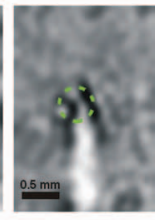

$29.0 \mathrm{~T}$

$-5140 \mathrm{~T}^{2} / \mathrm{m}$

$-0.15$
FIG. 1. (Color) Magnetically tuned gravity during crystal growth. (a) A growing crystal depletes its surrounding solution (1), leading to a growth plume (2) and convective flows (3). (b) At zero effective gravity convection is cancelled and the diffusion field expands. (c) In inverted gravity the buoyancy-driven convection is reversed, and a downward growth plume is formed. (d) The width of the depletion zone as function of $G_{\text {eff. }}$ [(e)-(i)] Experimental shadowgraphy images of a growing lysozyme crystal (indicated by the dashed green circles) in solution for $G_{\text {eff }}$ ranging from -0.15 to 1 .

camera. Variations in the concentration of the fluid result in local differences in the refractive index, which appear as intensity variations in the image. The sensitivity to concentration differences scales with the degree of being out of focus. ${ }^{15}$

We used HEWL from Sigma-Aldrich (Lot No. 094K1454), which was dissolved and dialysed against a $0.05 \mathrm{M} \mathrm{NaOAc} / \mathrm{HOAc}$ buffer solution of $p \mathrm{H} 4.5$ at room temperature before use. Tetragonal lysozyme crystals were grown from a solution of $30 \mathrm{mg} / \mathrm{ml} \mathrm{HEWL}, 0.685 \mathrm{M} \mathrm{NaCl}$, and $0.05 \mathrm{M} \mathrm{NaOAc} / \mathrm{HOAc}$ at $p \mathrm{H} 4.5$ and $18{ }^{\circ} \mathrm{C}$. Crystals were taken from the growth vessel and placed as a seed in the glass cuvette for the magnet experiments. The crystal was manipulated to be in the field of view of the insert, after which the solution was removed and the cuvette was placed in a refrigerator at $4{ }^{\circ} \mathrm{C}$ for $20 \mathrm{~min}$. As a result, the crystal is attached to the glass wall of the cuvette. Finally, the cuvette was refilled with the same solution as used during growth and placed in the insert for experiments.

The condition for convection damping is determined by $\alpha$ and $\beta$ in Eq.(1). For HEWL $\alpha$ is $0.303 \mathrm{~kg} \mathrm{~m}^{-3} / \mathrm{mg} \mathrm{ml}^{-1}$, ${ }^{16}$ and we have determined $\beta$ to be $(-1.2 \pm 0.5) \times 10^{-9} \mathrm{ml} / \mathrm{mg}$ using a magnetic susceptibility balance. Inserting these values in Eq. (1) we expect that convection is damped at $B_{z} B_{z}^{\prime}$ (a)
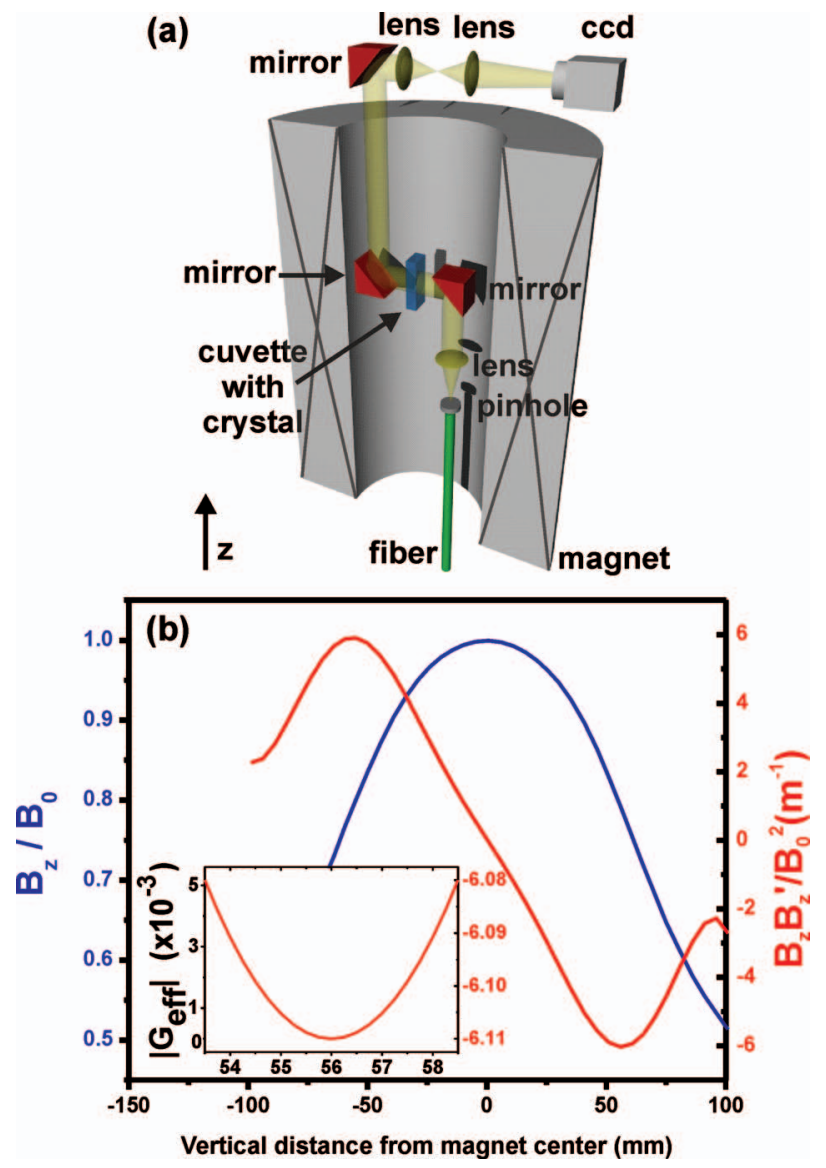

FIG. 2. (Color) Experimental setup for in situ observation of convective fluid flows in a $33 \mathrm{~T}$ magnet. (a) Schematic representation of the shadowgraphy insert used to visualize density variations in solutions. (b) Profiles of magnetic field and field times field gradient $\left(B_{z} B_{z}^{\prime}\right)$ scaled to a $B_{0}$ background field. The inset shows the $B_{z} B_{z}^{\prime}$ profile around the optimum position and the corresponding effective gravity.

$=-3100 \pm 1500 \mathrm{~T}^{2} / \mathrm{m}$, which is significantly larger than the $-1500 \mathrm{~T}^{2} / \mathrm{m}$ needed for simple levitation of the bulk solution. ${ }^{9}$ We determined the actual gradient field at which the growth plume disappears by using shadowgraphy. Figure 1(e) shows a growing HEWL crystal at zero field gradient, and the convection plume is clearly visible as a white streak rising upward from the crystal. In the picture the crystal itself is blurred because for shadowgraphy out-of-focus images have to be taken. The growth plume disappears, and thus convection is suppressed at a gradient magnetic field of $-4450 \pm 30 \mathrm{~T}^{2} / \mathrm{m}$ [Fig. 1(g)]. The value falls within our estimate using $\alpha$ and $\beta$, but is much higher than previously expected $^{5,10}$ and requires the largest magnets currently available. In fact, this value for the gradient field accurately determines $\beta$ as $(-0.84 \pm 0.06) \times 10^{-9} \mathrm{ml} / \mathrm{mg}$.

This result unambiguously shows that gradient magnetic fields can create conditions on earth that mimic those in space-based microgravity. Most importantly, however, is the fact that by changing the magnetic field strength the effective gravity for convection can be continuously varied. If we define ${ }^{17}$

$$
G_{\text {eff }}=1-\frac{\beta}{\alpha \mu_{0} g} B_{z} B_{z}^{\prime},
$$

$G_{\text {eff }}$ is expressed in terms of the earth's gravitational acceleration $g$. By varying the magnetic field we are able to 


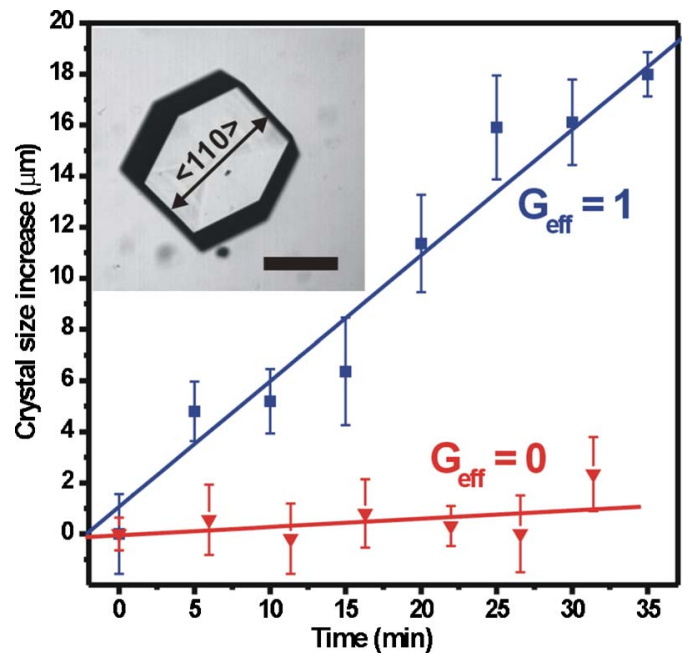

FIG. 3. Growth rate of tetragonal HEWL crystals at normal and zero effective gravity $G_{\text {eff. }}$ The squares (triangles) denote the increase in HEWL crystal size at a $G_{\text {eff }}$ of $1(0)$, obtained in the $\langle 110\rangle$ direction. The inset shows a tetragonal HEWL crystal similar to those used in the experiments and the $\langle 110\rangle$ direction with respect to the morphology.

change $G_{\text {eff }}$ and as a result the convection is tuned from normal, with a growth plume upwards [Figs. 1(e) and 1(f)], via cancellation at $G_{\text {eff }}=0[$ Fig. $1(\mathrm{~g})]$, to inverted with the growth plume downwards for negative values of $G_{\text {eff }}[$ Figs. 1(h) and 1(i)].

The range of field gradients at which convection is stopped is quite small, $\pm 30 \mathrm{~T}^{2} / \mathrm{m}$ centered around $-4450 \mathrm{~T}^{2} / \mathrm{m}$, which corresponds to $B=27 \mathrm{~T}$ in the magnet we used. Decreasing (increasing) the magnetic field by only $0.1 \mathrm{~T}\left(G_{\mathrm{eff}} \approx \pm 0.005\right)$ already results in appreciable convection and thus in upward (downward) growth plumes. This strong effect is caused by the steep dependence of the balance between convective flow and mass diffusion on $G_{\text {eff }}$, which is reflected by the thickness of the depletion zone. For example, Fig. 1(d) shows the theoretically calculated, and for $\mathrm{NiSO}_{4} \cdot 6 \mathrm{H}_{2} \mathrm{O}$ experimentally demonstrated, ${ }^{17}$ dependence of the thickness of the depletion zone $\delta$ on gravity. Since $\delta \propto\left|G_{\text {eff }}\right|^{-1 / 4}$ it diverges near zero, which implies that the field gradient has to be set quite precisely. Such a strong dependence also puts constraints on the spatial variation of $G_{\text {eff }}$ within a magnet. From Eq. (2) we calculate $G_{\text {eff }}$ as function of the position around the crystal using the experimental field profile [inset of Fig. 2(b)], which shows that changes of $G_{\text {eff }}$ over the relevant region are within \pm 0.005 . Despite the precise condition on the required field gradient, milligravity rather than microgravity ${ }^{18}$ is sufficient to make convective transport slower than that due to diffusion and to dampen convection.

To show that the suppression of convection indeed affects crystal growth, we have measured the growth rate of two lysozyme crystals, one at $\mathrm{G}_{\mathrm{eff}}=1$ and one at 0 , at otherwise identical conditions (Fig. 3). Here the same imaging setup was used, but now with the crystal in focus to determine the position of its surface. The growth rate drops a factor of 15 from $30 \pm 2$ to $2 \pm 2 \mu \mathrm{m} / \mathrm{h}$ when convection is stopped and the depletion zone is expanded, which is similar to results obtained under space-based microgravity. ${ }^{19}$

In contrast with other methods to suppress convection such as gel growth ${ }^{20,21}$ and microfluidics, ${ }^{22}$ gradient magnetic fields offer a powerful way to tune the effective gravity during crystal growth under earth-based conditions, with far easier access, availability, and including in situ observation. Especially for protein crystal growth this possibility is very attractive, since the tunability will allow the optimization of the crystal quality by finding the right balance between mass transport towards the crystal and the incorporation rate of molecules at the crystal surface. The required gradient magnetic fields for suppression of convection are found to be in the $4000-5000 \mathrm{~T}^{2} / \mathrm{m}$ range. Because density and susceptibility are closely related, we expect that this value is rather similar for most diamagnetic proteins. We foresee that our determination of the proper conditions for which convection is suppressed will trigger the design and construction of dedicated magnets that are capable of sustaining high field gradients for the several days that are needed to grow protein crystals.

The authors thank J. Rook, R. van Stijn, and Mrs. J. van Oostrum for technical support and D. van Heijnsbergen for a critical reading of the manuscript. This work is part of the research programs of the "Stichting voor Fundamenteel Onderzoek der Materie" (FOM), and the Council for the Chemical Sciences (CW), financially supported by the Netherlands Organisation for Scientific Research (NWO). One of the authors (K.T.) was supported by the Japan Space Forum as part of the "Ground Based Research Announcement for Space Utilization."

${ }^{1}$ E. H. Snell and J. R. Helliwel, Rep. Prog. Phys. 68, 799 (2005).

${ }^{2}$ A. Vergara, B. Lorber, C. Sauter, R. Giege, and A. Zagari, Biophys. Chem. 118, 102 (2005).

${ }^{3}$ W. Littke and C. John, Science 225, 203 (1984).

${ }^{4}$ D. Braithwaite, E. Beaugnon, and R. Tournier, Nature (London) 354, 134 (1991).

${ }^{5}$ N. Ramachandran and F. W. Leslie, J. Cryst. Growth 274, 297 (2005).

${ }^{6}$ P. W. G. Poodt, M. C. R. Heijna, K. Tsukamoto, W. J. de Grip, P. C. M. Christianen, J. C. Maan, W. J. P. van Enckevort, and E. Vlieg, Appl. Phys. Lett. 87, 214105 (2005).

${ }^{7}$ E. Beaugnon and R. Tournier, Nature (London) 349, 470 (1991).

${ }^{8}$ A. K. Geim, Phys. Today 51, 36 (1998).

${ }^{9}$ N. I. Wakayama, Cryst. Growth Des. 3, 17 (2003).

${ }^{10}$ N. I. Wakayama, Jpn. J. Appl. Phys., Part 2 44, L833 (2005).

${ }^{11}$ P. J. Shlichta, J. Cryst. Growth 76, 656 (1986).

${ }^{12}$ Y. Ikezoe, N. Hirota, J. Nakagawa, and K. Kitazawa, Nature (London) 393, 749 (1998).

${ }^{13}$ A. T. Catherall, L. Eaves, P. J. King, and S. R. Booth, Nature (London) 422, 579 (2003)

${ }^{14}$ A. McPherson, Crystallization of Biological Macromolecules (Cold Spring Harbor Laboratory, New York, 1999).

${ }^{15}$ G. Settles, Schlieren and Shadowgraphy Techniques (Springer, Berlin, 2001).

${ }^{16}$ W. J. Fredericks, M. C. Hammonds, S. B. Howard, and F. Rosenberger, J. Cryst. Growth 141, 183 (1994).

${ }^{17}$ P. W. G. Poodt, M. C. R. Heijna, K. Tsukamoto, W. J. de Grip, P. C. M. Christianen, J. C. Maan, W. J. P. van Enckevort, and E. Vlieg, Cryst. Growth Des. 6, 2275 (2006).

${ }^{18}$ N. Ramachandran, C. R. Baugher, and R. J. Naumann, Microgravity Sci. Technol. 8, 170 (1995).

${ }^{19}$ F. Otálora, J. M. García-Ruiz, L. Carotenuto, D. Castagnolo, M. L. Novella, and A. A. Chernov, Acta Crystallogr., Sect. D: Biol. Crystallogr. 58, 1681 (2002)

${ }^{20}$ M. C. Robert, K. Provost, and F. Lefaucheux, in Crystallization of nucleic acids and proteins: a practical approach, edited by A. Ducruix and R. Giegé (IRL, Oxford, 1999), p. 149.

${ }^{21}$ J. M. García-Ruiz, J. Drenth, M. Riés-Kautt and A. Tardieu, in A World without Gravity: Research in Space for Health and Industrial Processes, edited by G. Seibert, B. Titton, and B. Battrick (ESA, Noordwijk, The Netherlands, 2001), p. 159.

${ }^{22}$ D. C. Carter, P. Rhodes, D. E. McRee, L. W. Tari, D. R. Dougan, G. Snell, E. Abola, and R. C. Stevens, J. Appl. Crystallogr. 38, 87 (2005). 